\title{
Myocardial Segmentation of Area at Risk Based on Coronary Computed Tomography Angiography and Voronoi Diagram in Comparison with Magnetic Resonance Perfusion Imaging
}

\author{
Naoki Fukuyama1*, Teruhito Kido', Akira Kurata1, Yuki Tanabe1, Tomoyuki Kido1, \\ Takahiro Yokoi'1, Ryo Ogawa1, Hikaru Nishiyama1, Teruyoshi Uetani², Teruhito Mochizuki' \\ ${ }^{1}$ Department of Radiology, Ehime University Graduate School of Medicine, Toon, Japan \\ ${ }^{2}$ Department of Cardiology, Ehime University Graduate School of Medicine, Toon, Japan \\ Email: *n.fukuyama68@gmail.com
}

How to cite this paper: Fukuyama, N., Kido, T., Kurata, A., Tanabe, Y., Kido, T., Yokoi, T., Ogawa, R., Nishiyama, H., Uetani, T. and Mochizuki, T. (2017) Myocardial Segmentation of Area at Risk Based on Coronary Computed Tomography Angiography and Voronoi Diagram in Comparison with Magnetic Resonance Perfusion Imaging. Open Journal of Radiology, 7, 922.

https://doi.org/10.4236/ojrad.2017.71002

Received: January 24, 2017

Accepted: February 26, 2017

Published: March 1, 2017

Copyright @ 2017 by authors and Scientific Research Publishing Inc. This work is licensed under the Creative Commons Attribution International License (CC BY 4.0).

http://creativecommons.org/licenses/by/4.0/ (c) (i) Open Access

\begin{abstract}
Purpose: To assess the clinical feasibility of automated segmentation of the myocardial area at risk (MAAR) using coronary computed tomography angiography (CT-MAAR), as compared to stress magnetic resonance myocardial perfusion imaging (MR-MPI). Materials and Methods: Thirty patients who underwent coronary computed tomography angiography (CTA) and stress MR-MPI were retrospectively evaluated. The myocardial territory of the left ventricle (LV) distal to coronary artery stenosis $(\geq 50 \%$ or $\geq 70 \%$ stenosis on coronary CTA) was three-dimensionally quantified using a Voronoi diagram. The ratio of all stenosis-related territories to the LV volume was defined as CT-MAAR (\%-LV volume). The proportion of segments with perfusion defects in stress MR-MPI to the total of 16 segments (range: $0 \%-100 \%$; with a $6.3 \%$-interval scale) was defined as the reference. Correlation was assessed using Spearman's test. The capability of CT-MAAR to predict the ischemic burden was assessed. Results: Stress MR-MPI depicted a median ischemic burden of $25.2 \%$ (range: $18.9 \%-44.1 \%$ ) in 30 patients without myocardial infarction. When CTA stenosis criteria of $\geq 50 \%(n=30)$ and $\geq 70 \%(n=27)$ were applied to estimate CT-MAAR, the median CT-MAAR values were $48.2 \%(31.6 \%-64.3 \%)$ and $32.5 \%(23.7 \%-51.9 \%)$, respectively. The correlations between the CT-MAAR values and the MR-based ischemic burden were significant ( 0.73 and 0.97 for $\geq 50 \%$ and $\geq 70 \%$ stenosis, respectively). CTMAAR predicted the MR-based ischemic burden within \pm 1 segment of \%-LV $(6.3 \%)$ in $40 \%(12 / 30)$ of patients with $\geq 50 \%$ stenosis, and in $81.5 \%(22 / 27)$ of
\end{abstract}


patients with $\geq 70 \%$ stenosis. Conclusions: Comprehensive assessment of resting coronary CTA combined with Voronoi diagram-based myocardial segmentation may help predict the myocardial ischemic burden in patients with severe coronary CTA stenosis.

\section{Keywords}

Computed Tomography, Ischemia, Myocardial Area at Risk

\section{Introduction}

Coronary computed tomography angiography (CTA) is widely used in clinical practice for assessing obstructive coronary artery disease (CAD), because of its high sensitivity and negative predictive value [1] [2] [3]. However, because of the limited spatial resolution, further evaluation, such as invasive coronary angiography (ICA) or stress myocardial perfusion imaging (MPI), is often required in the diagnostic workflow of coronary artery disease (CAD) [4]. Stress MPI, using single-photon-emission computed tomography (SPECT) and magnetic resonance imaging (MR), can non-invasively detect and quantify ischemic myocardium as a standard reference [5] [6], and is useful as the ischemic ratio of the left ventricular (LV) myocardium provides important information for diagnostic and therapeutic purposes in patients with CAD [7].

Furthermore, the Voronoi diagram is a type of centerline method that divides space (i.e., volume) by seeded points or lines [8], and is utilized in industry and geography. In diagnostic imaging, a previous study has shown the usefulness of this algorithm for liver segmentation, based on CT portal venography [9], and a recent study has reported that ICA-based stenosis-related CT myocardial territory correlates with the SPECT-based myocardial area at risk (MAAR) [10]. Coronary CTA stenosis-related CT myocardial territory is an assumption of the maximum MAAR that is obtained from a resting coronary CTA dataset. Stress MR-MPI has a high spatial resolution and yields better diagnostic performance in assessing multi-vessel disease in comparison with SPECT-MPI [11] [12]. Thus, this study aimed to assess the clinical feasibility of applying automated segmentation of MAAR using coronary CTA, as compared to stress MR-MPI.

\section{Materials and Methods}

\subsection{Study Design}

This retrospective observational study was approved by the local ethics committee (clinical research 1509002). The need for informed consent was waived because of the retrospective nature of the study. From June 2009 to March 2016, we retrospectively collected the records of 91 consecutive patients who underwent coronary CTA and stress MR-MPI within an interval of less than 6 months. All patient information was protected in compliance with the guidelines pro- 
vided by the institutional review board. The flow chart for patient selection is shown in Figure 1. Patients with myocardial ischemia diagnosed by stress MRMPI were excluded if they fulfilled the following criteria: 1) no invasive coronary angiography (ICA) available; 2) previous history of revascularization therapy, such as coronary artery bypass grafting $(\mathrm{CABG})$ or percutaneous coronary intervention (PCI); 3) heart valve disease; 4) cardiomyopathy; 5) total coronary artery occlusion confirmed byICA and 6) poor image quality of coronary CTA.

\subsection{Coronary CTA}

We used a 256-slice (128 multi-detector row) CT (Brilliance iCT; Philips Healthcare, Cleveland, OH, USA) and an automatic dual-head injector (Stellant Dual Flow; Nihon MEDRAD K.K., Osaka, Japan). All patients received $1.2 \mathrm{mg}$ (i.e., 2 puffs) of sublingual nitroglycerin (Myocor spray; Astellas Pharma, Tokyo, Japan). For patients with a resting heart rate of more than 60 beats/min, an intravenous beta-blocker $(0.125 \mathrm{mg} / \mathrm{kg}$ of landiolol hydrochloride, Corebeta; Ono Pharmaceutical Co., Osaka, Japan) was administered 5 min before the timing-bolus scan, to reduce the heart rate. Coronary CTA was performed with iohexol (Omnipaque; $350 \mathrm{mg}$ iodine/mL; Daiichi Sankyo, Tokyo, Japan) or with iopamidol (Iopamiron; $370 \mathrm{mg}$ iodine/mL; Bayer Yakuhin, Osaka, Japan) at an injection rate of $5.0-5.5 \mathrm{~mL} / \mathrm{s}$ for $10 \mathrm{~s}$, followed by a saline chaser $(20 \mathrm{~mL}, 5.0$ $5.5 \mathrm{~mL} / \mathrm{s}$ ). The scan parameters were as follows: retrospective electrocardiogramgated mode (heart rate $>60$ beats/min) or prospective electrocardiogram-gated mode (heart rate $\leq 60$ beats $/ \mathrm{min}$ ); tube voltage, $120 \mathrm{kV}$; effective tube current time-product, 800 - $1300 \mathrm{mAs} /$ rotation with dose modulation; gantry rotation

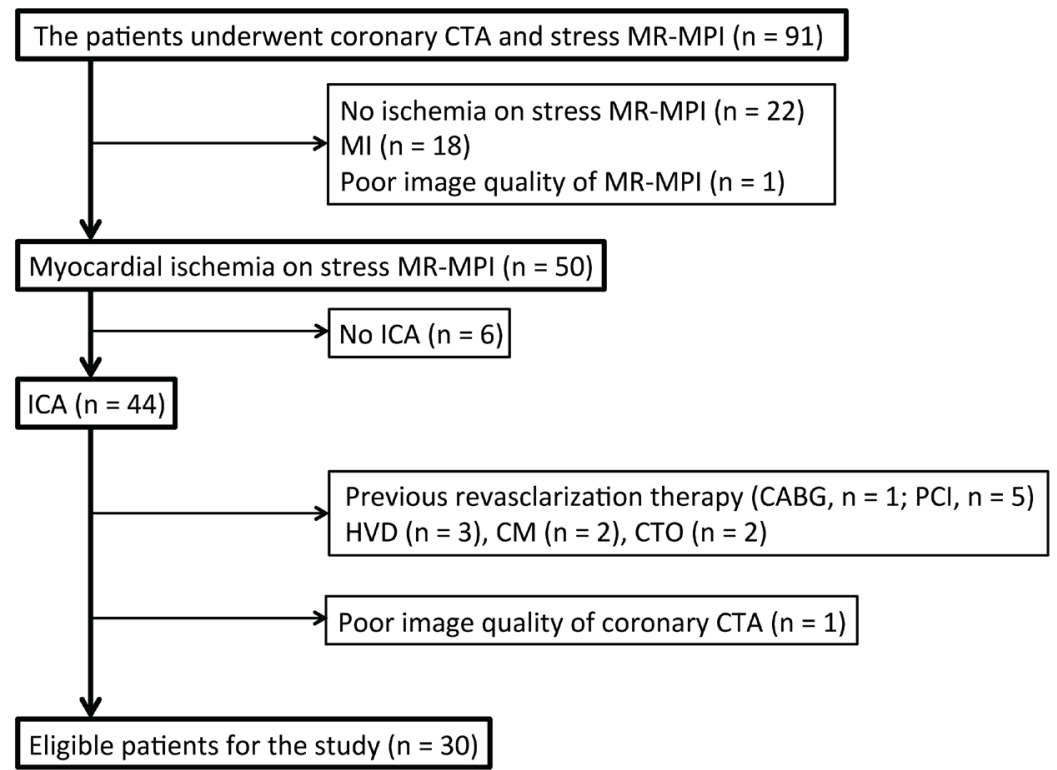

Figure 1. Flowchart of the study participants. CABG, coronary artery bypass grafting; $\mathrm{CM}$, cardiomyopathy; CTA, computed tomography angiography; CTO, total coronary artery occlusion; HVD, heart valve disease; MI, myocardial infarction; ICA, invasive coronary angiography; MR-MPI, magnetic resonance myocardial perfusion imaging; PCI, percutaneous coronary intervention. 
time, 0.27 s/rotation; collimation, $2 \times 128 \times 0.625 \mathrm{~mm}$ with a dynamic $z$-focal spot; 250-mm display field of view; 0.8/0.4-mm slice thickness/overlap; and 512 $\times 512$ image matrix. Image reconstruction was performed individually to reduce motion artifacts, with $0.8-\mathrm{mm}$ slice thickness and $0.4-\mathrm{mm}$ intervals using hybrid iterative reconstruction (iDose $\mathrm{e}^{4}$ level 4; Philips Healthcare, Cleveland, OH, USA) and a medium-smooth cardiac kernel.

\subsection{Stress Cardiac MR-MPI}

We used a 3-T MR system (Achieva 3.0 T Quasar Dual; Philips Healthcare, Cleveland, OH, USA) equipped with a 32-element cardiac phased-array coil. Using an established comprehensive cardiac MR protocol [13], stress and rest MR perfusion and late images were obtained. For the stress image, pharmacological hyperemia was induced with an adenosine triphosphate infusion $(0.16$ $\mathrm{mg} \cdot \mathrm{min}^{-1} \cdot \mathrm{kg}^{-1}$ ) for $\geq 3 \mathrm{~min}$. Stress and resting MR perfusion images (for $35 \mathrm{~s}$ ) were obtained from three short-axis images of the basal, mid-, and apical LV, using a two-dimensional (2D) T1 turbo field-echo sequence with k-space and time broad-use linear acquisition speed-up technique (k-t BLAST), after the injection of gadopentetate dimeglumine $(0.05 \mathrm{mmol} / \mathrm{kg}$; Magnevist; Schering, Berlin, Germany), followed by a $30-\mathrm{mL}$ saline chaser, at an injection rate of $4 \mathrm{~mL} / \mathrm{s}$. Late images were obtained $10 \mathrm{~min}$ after the resting image using an inversionrecovery $3 \mathrm{D} \mathrm{T} 1$ turbo field-echo sequence. The inversion time was individually determined using the Look-Locker sequence.

\subsection{Invasive Coronary Angiography}

ICA was performed following the standard institutional catheterization approach. Quantitative coronary analysis was performed by an independent cardiologist (T.U., 15 years of experience), who was blinded to other results, using commercially available software (CAAS5.9; Pie Medical Imaging, Maastricht, the Netherlands). Coronary artery stenosis $\geq 50 \%$ and $\geq 70 \%$ were considered as significant and obstructive CAD, respectively. When multiple stenoses were seen in two or more segments, the proximal stenosis was defined as the culprit stenosis. All coronary artery segments and stenotic lesions ( $\geq 50 \%$ stenosis) were classified by the main coronary vessels: the left anterior descend- ing artery (LAD), including diagonal branches, the left circumflex artery (LCX), including the high lateral branch and ramus intermedius, and the right coronary artery (RCA), based on the American Heart Association guidelines [14].

\subsection{MR-Based Ischemic Burden}

The 16-segment model, excluding the apex, was applied [15]. The perfusion images were visually analyzed by two observers (T.K. and R.O., with 3 years and 8 years of experience in cardiac MR, respectively). These observers were blinded to all other data. If a perfusion defect in the LV myocardium was present in four or more cardiac cycles in the stress image, but not present in the resting image, it was considered to be a stress-induced perfusion defect [16]. When a segment 
with $\geq 50 \%$ of stress-induced perfusion defect was seen along the circumferential direction of the LV short axial image, corresponding to significant ICA stenosis by vessel-based analysis, it was defined as the ischemic segment in this study. Discrepancies between the opinions of the first two observers were solved by a third senior observer (T. K., with 15 years' experience).In the late image, a segment with any late gadolinium enhancement was defined as a segment with myocardial infarction, irrespective of the presence or absence of myocardial ischemia. Patients with any segments showing late gadolinium enhancement were excluded from the study. The ratio of the number of segments with myocardial ischemia to the total of 16 segments was defined as the standard reference in this study (range: $0 \%-100 \%$ with a $6.3 \%$-interval scale). The vesselbased analysis of the 16-segment model was based on a standardized myocardial segmentation [15].

\subsection{Assessment of Coronary CTA}

All CTA images were evaluated by two observers (Y.T. and T.Y., who had 4 years and 2 years of experience in cardiac CT, respectively) using a commercially available workstation (Aquarius intuition; TERARECON, Inc., Tokyo, Japan). First, an overall assessment of image quality was performed at the subject level to assess misalignment of the coronary arteries between the slabs and discontinuity of coronary vessels due to motion artifacts and arrhythmia. If any of the aforementioned requirements were unsuitable, the patient was excluded from the study. Using a single dataset of coronary CTA with sufficient image quality, stenosis severity per segment was semiquantitatively assessed using the standard guidelines for reporting coronary CTA [17]: "non-CAD", 0\% - 49\%; "moderate", 50\% - 69\%; "severe", 70\% - 99\%; and "occluded", 100\%. The unassessable calcified segments were assumed to indicate severe stenosis. When multiple stenoses were found in the same coronary segment and vessel, the proximal stenosis was considered to be the culprit stenosis. Discrepancies were solved by consensus. Coronary artery segmentation was applied, in addition to ICA.

\subsection{CTA-Based MAAR}

The same two observers evaluated the coronary CTA-based myocardial territory using dedicated software (TVA; TERARECON Inc. Tokyo, Japan). Post- processing of Voronoi diagram-based myocardial segmentation using coronary CTA was shown in Figure 2. In a series of Voronoi diagram-based myocardial segmentations based on a single CT dataset, the LV myocardium was automatically extracted using basic cardiac function analysis, and the coronary arteries were extracted in a semiautomatic manner. The software three-dimensionally integrated the two datasets and quantified the LV territories distal to any point on coronary CTA irrespective of coronary stenosis severity.

For this study, the stenosis-related CT myocardial territory was quantified at both levels of stenosis ( $\geq 50 \%$ and $\geq 70 \%$ on coronary CTA). The discrepancies of the stenosis-related CT myocardial territories were individually reviewed and 


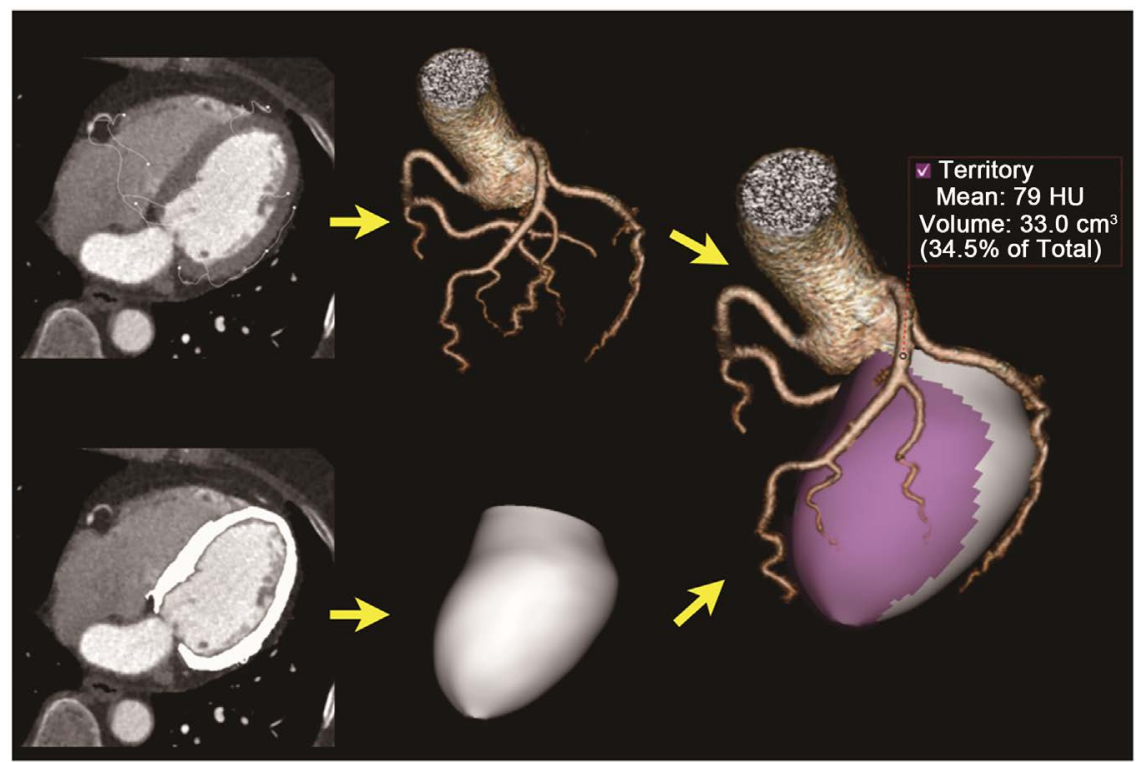

Figure 2. Post-processing of Voronoi diagram-based myocardial segmentation using coronary computed tomography (CT) angiography. From a single dataset of cardiac CT, coronary arteries and left ventricular myocardium are removed and integrated in the dedicated software. The dependent myocardial territory to a point on coronary CT angiography is three-dimensionally quantified.

solved by consensus. The two sums of all stenosis-related CT myocardial territories (i.e., the ratio of the LV volume at risk to the whole $\mathrm{LV}$ volume) were calculated using the two standards of the CTA stenosis ( $\geq 50 \%$ and $\geq 70 \%$ ) as significant and obstructive CTA stenosis-based CT-MAAR, respectively.

\subsection{Statistical Analysis}

Categorical variables were expressed as proportions and continuous variables were Altman plotting expressed as the mean \pm the standard deviation or as the median (interquartile range), as appropriate. With regard to coronary CTA stenosis severity and the stenosis-related CT myocardial territory for stenotic lesions $\geq 50 \%$, the intra- and inter-observer reproducibility of the two operators were assessed using Spearman's test and Bland-Altman plotting.

Correlations between CTA-based MAAR and MR-based ischemic burden were evaluated with Spearman's test. The ability of CTA-based MAAR to estimate the MR-based ischemic burden within $\pm 6.3 \%$, corresponding to a single segment of the LV $(100 / 16=6.3)$ in the assessment of MR-based ischemia, was calculated. All analyses were performed using JMP version 11 (SAS Institute, Cary, NC, USA). For all analyses, $p<0.05$ was significant.

\section{Results}

\subsection{Patient Characteristics}

Of the 50 patients, 20 were excluded for the following reasons: no ICA $(n=6)$, previous revascularization therapy (CABG, $n=1$; PCI, $n=5$ ), valvular heart 
disease $(\mathrm{n}=3)$, cardiomyopathy $(\mathrm{n}=2)$, total coronary artery occlusion $(\mathrm{n}=2)$, and poor image quality on MR-MPI $(n=1)$ or on coronary CTA $(n=1)$. Thirty patients were finally analyzed (24 men and 6 women; mean age, $67.5 \pm 7.9$ years). The patient characteristics are listed in Table 1. ICA revealed 60 coronary artery lesions with $>50 \%$ stenosis and 28 coronary artery lesions with $>70 \%$ stenosis. The numbers of diseased vessels were 29 in the LAD, 18 in the LCX, and 13 in the RCA, respectively. The ICA-based diagnosis was single-vessel disease $(\mathrm{n}=16)$, double-vessel disease $(\mathrm{n}=12)$, and triple-vessel disease $(\mathrm{n}=2)$. The median MR-based ischemic burden was $25.2 \%$ (18.9\% - 44.1\%).

\subsection{Coronary CTA Analysis}

A total of 438 segments were assessed. The intra- and inter-observer agreement for the semiquantitative assessment of stenotic severity on coronary CTA was 0.71 and 0.67 , respectively. The by-consensus diagnosis per segment was as follows: non-CAD stenosis $(\mathrm{n}=361)$, moderate stenosis $(\mathrm{n}=30)$, severe stenosis ( $\mathrm{n}$ $=28)$, occluded lesions $(\mathrm{n}=3)$, and unassessable calcified segments $(\mathrm{n}=16)$. When using the CTA stenosis criterion of $\geq 50 \%$, 77 lesions in 30 patients met the criteria for significant CTA-based MAAR. However, when using the CTA stenosis criterion of $\geq 70 \%, 47$ lesions in 27 patients met the criteria for obstructive CTA-based MAAR.

\subsection{Stenosis-Related CT Myocardial Territory}

Seventy-seven coronary CTA lesions with a stenosis of $\geq 50 \%$ were assessed. Voronoi-based segmentation was successfully performed for all lesions. The respesentati ve for Compute cases are shown in Figure 3 and Figure 4. The intra- and

Table 1. Patient characteristics.

\begin{tabular}{cc}
\hline & $\mathrm{N}=30$ \\
\hline Male/female & $24(80.0 \%) / 6(20.0 \%)$ \\
Age (years) & $67.5 \pm 7.9$ \\
Hypertension & $13(43.3 \%)$ \\
Hyperlipidemia & $14(46.7 \%)$ \\
Diabetes mellitus & $8(26.7 \%)$ \\
Smoking & $12(40.0 \%)$ \\
Family history of CAD & $8(26.7 \%)$ \\
Time between coronary CTA and MR-MPI (days) & $29(11.5-49)$ \\
Calcium score (Agatston score) & $405.4(95.4-1031.8)$ \\
${ }^{*}$ Number of diseased vessels confirmed with ICA & $46 / 90$ \\
${ }^{*}$ Single-vessel disease & 16 \\
${ }^{*}$ Double-vessel disease & 12 \\
${ }^{*}$ Triple-vessel disease & 2 \\
\hline
\end{tabular}

$\mathrm{CAD}=$ coronary artery disease; $\mathrm{CTA}=$ computed tomography angiography; MR-MPI = magnetic resonance myocardial perfusion imaging; ICA = invasive coronary angiography; presented as $\mathrm{N}(\%)$, mean $\pm \mathrm{SD}$ or median (interquartile range), unless otherwise stated. ${ }^{\star}$ Invasive coronary angiography stenosis of $\geq 50 \%$ are significant stenosis. 


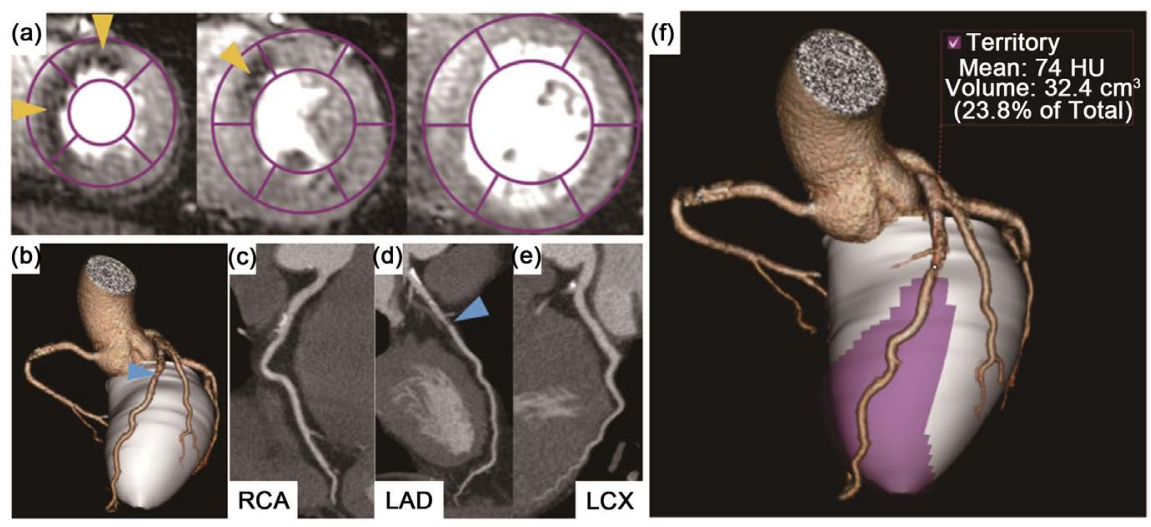

Figure 3. Dataset of a 59-year-old man with effort angina. (a) Stress magnetic resonance myocardial perfusion imaging (MR-MPI) shows perfusion defects in the anteroseptal segments (yellow arrowhead). The MR-MPI-based myocardial ischemic burden was estimated to be $18.9 \%$; (b)-(e) Coronary computed tomography (CT) angiography shows single-vessel disease with severe stenosis in the left anterior descending coronary artery (LAD, blue arrowhead); (f) The CT-based myocardial area at risk using Voronoi diagram-based myocardial segmentation was $23.8 \%$. RCA, right coronary artery; LCX, left circumflex artery.
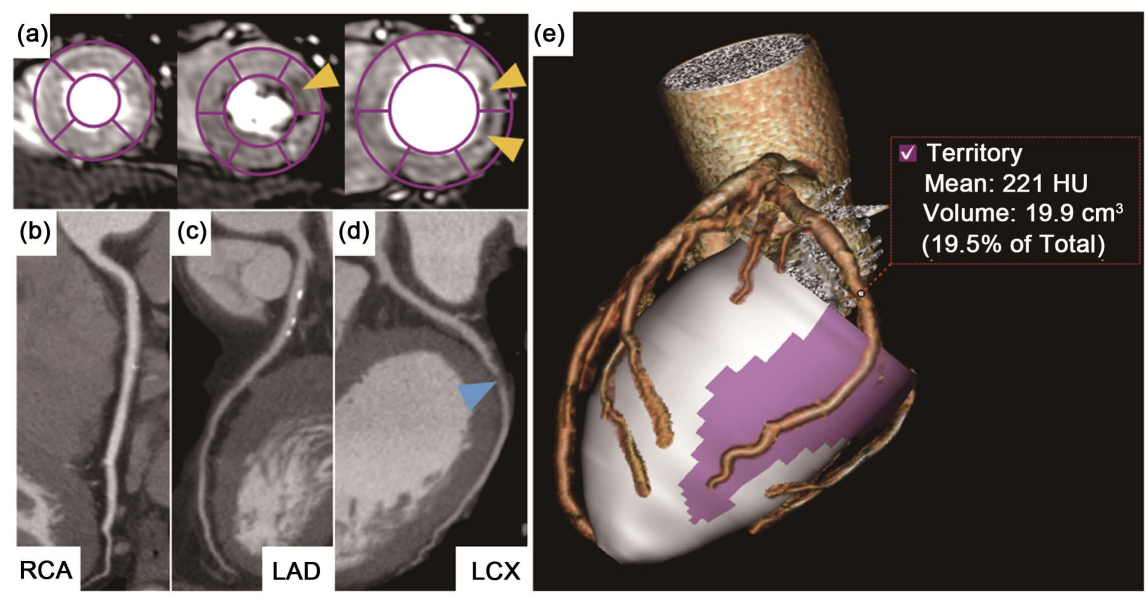

Figure 4. Dataset of a 71-year-old man with effort angina. (a) Stress magnetic resonance myocardial perfusion imaging (MR-MPI) demonstrates defects in the lateral segments (yellow arrowhead). The MR-MPI-based myocardial ischemic burden was estimated to be 18.9\%; (b)-(d) Coronary computed tomography (CT) angiography shows single-vessel disease with a severe stenosis in the left circumflex (LCX, blue arrowhead); (e) The CT-based myocardial area at risk was quantified as 19.5\%. LAD, left anterior descending coronary artery; RCA, right coronary artery.

inter-observer agreements of the significant CTA-related myocardial territories were 0.99 and 0.99 , respectively. The mean differences for intra- and inter-observer measurements of CT-based MAAR were $0.004 \%$ (95\% confidence interval [CI],$-0.053 \%$ to $0.045 \%$ ) and -0.003 (95\% CI, $-0.030 \%$ to $0.024 \%$ ), respectively.

Using the standard of $\geq 50 \%$ CTA stenosis, the median value of significant CTA-based MAAR was 48.2\% (range: 31.6\% - 64.3\%). Marked CTA stenosisbased CT-MAAR correlated with MR-based ischemic burden statistically significantly $(\mathrm{r}=0.73 ; p<0.001)$ (Figure $5(\mathrm{a})$ ). CT-MAAR predicted the ischemic 


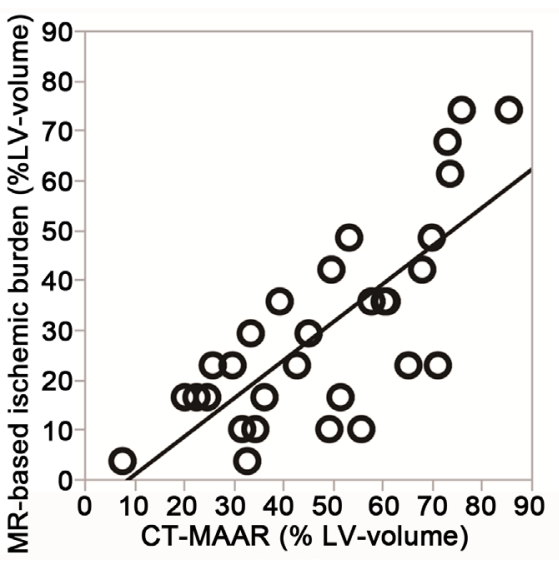

(a)

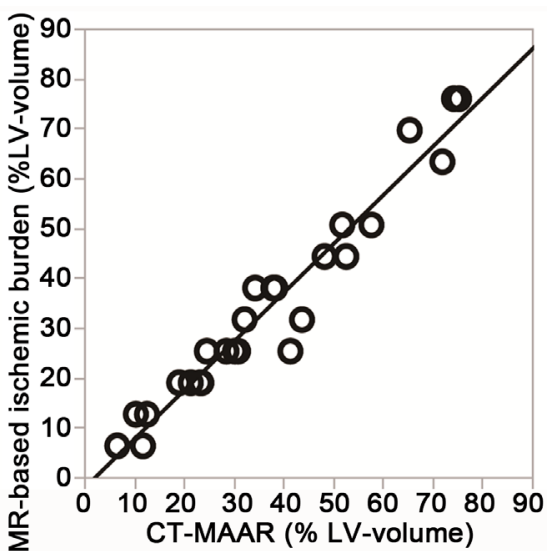

(b)

Figure 5. (a) and (b) The relationship and difference between myocardial area at risk assessed using computed tomography (CT-MAAR) and the magnetic resonance myocardial perfusion imaging (MR-MPI)-based ischemic burden. For the standard computed tomography angiography (CTA) stenosis criterion of $\geq 50 \%$, the correlation coefficient was 0.73 $(p<0.001)$. For the standard CTA stenosis criterion of $\geq 70 \%$, the correlation coefficient was $0.97(p<0.001)$. CTA, computed tomography angiography; LV, left ventricle; \%LV, the ratio of all stenosis-related territories to the left ventricle myocardial volume.

burden as assessed with MR to within $\pm 6.3 \%$ in $40 \%$ (12/30) of patients, but overestimated the area at risk by $6.3 \%$ in $60 \%(18 / 30)$ patients.

Using the standard of $\geq 70 \%$ CTA stenosis, the median value of obstructive CTA stenosis-based CT-MAAR was 32.5\% (range: 23.7\% - 51.9\%). The correlation between obstructive CTA stenosis-based CT-MAAR and MR-based ischemic burden was statistically significant $(r=0.97, p<0.001)$ (Figure $5(\mathrm{~b})$ ). CTA stenosis-based MAAR could predict the ischemic burden within $\pm 6.3 \%$ of that assessed using stress MR-MPI in 22 patients (81.5\%) and overestimated the area at risk by $6.3 \%$ in 5 patients $(18.5 \%)$.

\section{Discussion}

In this study, we showed that: 1) the reproducibility of the procedural steps for assessment of the CTA-based MAAR was good, and that 2) severe coronary CTA stenosis-based MAAR more accurately estimated the MR-based myocardial ischemic burden than did moderate stenosis.

Stress MR-MPI has been established as a useful diagnostic tool for detecting obstructive CAD [11]. The high spatial resolution of MR-MPI enables the delineation of subendocardial ischemia and myocardial infarction in patients with CAD [18]. Therefore, a comprehensive cardiac protocol involving the use of stress MR-MPI is helpful for diagnosing and managing CAD, even in patients with multivessel disease [19]. Stress MPI is essential for assessing the MAAR; therefore, many researchers have investigated stress-induced myocardial perfusion abnormalities using SPECT, MR, and CT [20] [21] [22].

By using a Voronoi diagram, CTA-based myocardial segmentation can indicate the theoretical maximum potential myocardial territory to a seeded point on the coronary CT angiogram. A recent study has demonstrated the high reprodu- 
cibility of CTA-based LV myocardial territory and the good correlation of CTbased MAAR with SPECT-based MAAR [10]. SPECT has been established as a clinical standard because of the substantial evidence supporting its use in the diagnosis and management of CAD [5] [20] [23]. However, some studies [11] [12] have discussed the potential disadvantages of SPECT as compared to MR-MPI, such as its lower spatial resolution and visualization of relative myocardial perfusion abnormalities.

Some researchers have reported clinical application of Voronoi diagrams in cardiovascular imaging [24] [25] [26]. Termeer et al. [25] utilized the Voronoi algorithm for estimating the MAAR on a 2D bull's eye map of the 17-segment model. Kang et al. [26] agreements of recently reported that the impact of coronary stenosis severity as assessed by intravascular ultrasound on the dependent myocardial territory is related to the severity of the invasive fractional flow reserve.

We considered that the CTA stenosis-based CT MAAR might contain some artificial post-processing. The study evaluated the intra- and inter-observer agreements of the CTA stenosis semi-quantification and (0.71 and 0.67, respectively) and those of the stenosis-related myocardial segmentation (0.99 and 0.99, respectively). These may indicate that the former ispotentially variable, while the stenosis-based myocardial segmentation on CT scans is highly reproducible, although the region of interest for coronary CTA stenosis was manually placed. A previous study reported the relationship between the extent of the severity of coronary artery stenosis on CT to myocardial ischemia as assessed with stress SPECT-MPI [27]; however, our study revealed the clinical significance of the CTA-based MAAR, according to coronary artery stenosis severity, by quantifying the individual stenosis-related myocardial territory, using the MR-based ischemic burden as the reference. In clinical practice, massive calcification, coronary stents, and intermediate stenosis remain a challenge for coronary CTA [2] [28]. The present study may show the additional value of this method for further examinations, such as stress MPI and invasive examination in the assessment of myocardial ischemia.

The present study has several limitations. First, the study population was relatively small. Second, stress MR-MPI as a reference was obtained with three cardiac short axial images, assessed using a conventional 16-segment model (excluding the apex), with a step-formed ordinal scale per 6.3\%, independent from the morphological volume-based or the transmural extent-based evaluation. It is to be noted that these differences between CT-MAAR and stress MR-MPI might potential place a limitation on the evaluation of CT-MAAR in the study. Third, this study excluded patients with myocardial infarction because a myocardial scar may influence the quantification of CT-MAAR, by affecting the volumetric estimation of CT-MAAR, and the true extent of myocardial ischemia. Fourth, the CT-MAAR was estimated based on the anatomical location of coronary stenosis, independent of whether the lesion was diagnosed as hemodynamically significant. Fifth, the precision of the ischemic burden in stress MR-MPI was not 
objectively validated on anatomical territory that corresponded to coronary artery stenosis. Further studies with a large number of patients will be required to clarify the significance of these findings in clinical practice.

\section{Conclusion}

Voronoi diagram-based myocardial segmentation has the potential for predicting the MAAR in patients with obstructive coronary stenosis on CTA. The estimation of MAAR using resting coronary CTA may provide useful information in clinical decision-making.

\section{References}

[1] Vanhoenacker, P.K., Heijenbrok-Kal, M.H., Van Heste, R., Decramer, I., Van Hoe, L.R., Wijins, W. and Hunink, M.G. (2007) Diagnostic Performance of Multidetector CT Angiography for Assessment of Coronary Artery Disease: Meta-Analysis. Radiology, 244, 419-428. https://doi.org/10.1148/radiol.2442061218

[2] Budoff, M.J., Dowe, D., Jollis, J.G., Gitter, M., Sutherland, J., Halamert, E., Scherer, M., Bellinger, R., Martin, A., Benton, R., Delago, A. and Min, J.K. (2008) Diagnostic Performance of 64-Multidetector Row Coronary Computed Tomographic Angiography for Evaluation of Coronary Artery Stenosis in Individuals without Known Coronary Artery Disease: Results from the Prospective Multicenter ACCURACY (Assessment by Coronary Computed Tomographic Angiography of Individuals Undergoing Invasive Coronary Angiography) Trial. Journal of the American College of Cardiology, 52, 1724-1732. https://doi.org/10.1016/j.jacc.2008.07.031

[3] Hoffmann, M.H., Shi, H., Schmitz, B.L., Schmid, F.T., Lieberknecht, M., Schulze, R., Ludwing, B., Kroschel, U., Jahnke, N., Haerer, W., Brambs, H.J. and Aschoff, A.J. (2005) Noninvasive Coronary Angiography with Multi-Slice Computed Tomography. JAMA, 293, 2471-2478. https://doi.org/10.1001/jama.293.20.2471

[4] Yamashina, A., Ueshima, K., Kimura, K., Kuribayashi, Y., Sakuma, H., Tamaki, N., Yoshida, K., Kitagawa, K., Kosuga, M., Otsuji, Y., Kihara, Y., Jinzaki, M., Chikamori, T., Teraoka, K., Hayashida, A., Harada, M., Yoshioka, K., Yoshinaga, K., Watanabe, N., Nishimura, S., Mizuno, K. and Yoshino, H.(2009) Guidelines for Noninvasive Diagnosis of Coronary Artery Lesions (JCS 2009). Circulation Journal, 73, 1019-1089.

[5] Hachamovitch, R., Berman, D.S., Kiat, H., Cohen, I., Cabico, J.A., Friedman, J. and Diamond, G.A. (1996) Exercise Myocardial Perfusion SPECT in Patients without Known CAD: Incremental Prognostic Value and Use in Risk Stratification. Circulation, 93, 905-914. https://doi.org/10.1161/01.CIR.93.5.905

[6] Nandalur, K.R., Dwamena, B.A., Choudhri, A.F., Nandalur, M.R. and Carlos, R.C. (2007) Diagnostic Performance of Stress Cardiac Magnetic Resonance Imaging in the Detection of Coronary Artery Disease: A Meta-Analysis. Journal of the American College of Cardiology, 50, 1343-1353. https://doi.org/10.1016/j.jacc.2007.06.030

[7] Hachamovitch, R., Hayes, S.W., Friedman, J.D., Cohen, I. and Berman, D.S. (2003) Comparison of the Short-Term Survival Benefit Associated with Revascularization Compared with Medical Therapy in Patients with No Prior Coronary Artery Disease Undergoing Stress Myocardial Perfusion Single Photon Emission Computed Tomography. Circulation, 107, 2900-2907. https://doi.org/10.1161/01.CIR.0000072790.23090.41

[8] Guibas, L. and Stolfi, J. (1985) Primitives for the Manipulation of General Subdivisions and the Computations of Voronoi Diagrams. ACM Transactions on Graphics, 
4, 74-123. https://doi.org/10.1145/282918.282923

[9] Debarba, H.G., Zanchet, D.J., Fracaro, D., Maciel, A. and Kalil, A.N. (2010) Efficient Liver Surgery Planning in 3D Based on Functional Segment Classification and Volumetric Information. Annual International Conference of the IEEE Engineering in Medicine and Biology Society, Buenos Aires, 31 August-4 September 2010, 47974800.

[10] Kurata, A., Kono, A., Sakamoto, T., Kido, T., Mochizuki, T., Higashino, H., Abe, M., Coenen, A., Saru-Chelu, R.G., Feyter, P.J., Krestin, G.P. and Nieman, K. (2015) Quantification of the Myocardial Area at Risk Using Coronary CT Angiography and Voronoi Algorithm-Based Myocardial Segmentation. European Radiology, 25, 49-57. https://doi.org/10.1007/s00330-014-3388-2

[11] Ishida, N., Sakuma, H., Motoyasu, M., Okinaka, T., Isaka, N., Nakano, T. and Takeda, K. (2003) Noninfarcted Myocardium: Correlation between Dynamic FirstPass Contrast-Enhanced Myocardial MR Imaging and Quantitative Coronary Angiography. Radiology, 229, 209-216. https://doi.org/10.1148/radiol.2291021118

[12] Greenwood, J.P., Maredia, N., Younger, J.F., Brown, J.M., Nixon, J., Everett, C.C., Bijsterveld, P., Ridgway, J.P., Radigenovic, A., Dickinson, C.J., Ball, S.G. and Plein, S. (2012) Cardiovascular Magnetic Resonance and Single-Photon Emission Computed Tomography for Diagnosis of Coronary Heart Disease (CE-MARC): A Prospective Trial. The Lancet, 379, 453-460. https://doi.org/10.1016/S0140-6736(11)61335-4

[13] Kramer, C.M., Barkhausen, J., Flamm, S.D., Kim, R.F. and Nagel, E. (2013) Society for Cardiovascular Magnetic Resonance Board of Trustees Task Force on Standardized Protocol. Standardized Cardiovascular Magnetic Resonance (CMR) Protocols 2013 Update. Journal of Cardiovascular Magnetic Resonance, 8, 15-91.

[14] Austen, W.G., Edwards, J.E., Frye, R.L., Gensini, G.G., Gott, V.L., Griffith, L.S., McGoon, D.C., Murphy, M.L. and Roe, B.B. (1975) A Reporting System on Patients Evaluated for Coronary Artery Disease. Report of the Ad Hoc Committee for Grading of Coronary Artery Disease, Council on Cardiovascular Surgery, American Heart Association. Circulation, 51, 5-40. https://doi.org/10.1161/01.CIR.51.4.5

[15] Cerqueira, M.D., Weissman, N.J., Dilsizian, V., Jacobs, A.K., Kaul, S., Laskey, W.K., Pennell, D.J., Rumberger, J.A., Ryan, T. and Verani, M.S. (2002) American Heart Association Writing Group on Myocardial Segmentation and Registration for Cardiac Imaging. Standardized Myocardial Segmentation and Nomenclature for Tomographic Imaging of the Heart. A Statement for Healthcare Professionals from the Cardiac Imaging Committee of the Council on Clinical Cardiology of the American Heart Association. Circulation, 105, 539-542.

https://doi.org/10.1161/hc0402.102975

[16] Jogiya, R., Morton, G., De Silva, K., Reyes, E., Hachamovitch, R., Kozerke, S., Nagel, E., Underwood, S.R. and Plein, S. (2014) Ischemic Burden by 3-Dimensional Myocardial Perfusion Cardiovascular Magnetic Resonance Comparison with Myocardial Perfusion Scintigraphy. Circulation: Cardiovascular Imaging, 7, 647-654.

https://doi.org/10.1161/CIRCIMAGING.113.001620

[17] Leipsic, J., Abbara, S., Achenbach, S., Cury, R., Earls, J.P., Mancini, G.J., Nieman, K., Pontone, G. and Raff, G.L. (2014) SCCT Guidelines for Interpretation and Reporting of Coronary CT Angiography: A Report of the Society of Cardiovascular Computed Tomography Guidelines Committee. Journal of Cardiovascular Computed Tomography, 8, 342-358. https://doi.org/10.1016/j.jcct.2014.07.003

[18] Gerbaud, E., Cochet, H., Bullier, E., Ragot, C., Gibert, S.H., Douard, H., Pucheu, Y., Laurent, F., Coste, P., Bordenave, L. and Montaudon, M. (2014) Peri-Infarct Ischemia Assessed by Cardiovascular MRI: Comparison with Quantitative Perfusion Sin- 
gle Photon Emission CT Imaging. The British Journal of Radiology, 87, 20130774. https://doi.org/10.1259/bjr.20130774

[19] Schwitter, J., Wacker, C.M., Wilke, N., Al-Saadi, N., Sauer, E., Huettle, K., Schonberg, S.O., Debl, K., Strohm, O., Ahlstrom, H., Dill, T., Hoebel, N. and Simor, T. (2012) MR-IMPACT Investigators. Superior Diagnostic Performance of Perfusion-Cardiovascular Magnetic Resonance versus SPECT to Detect Coronary Artery Disease: The Secondary Endpoints of the Multicenter Multivendor MR-IMPACT II (Magnetic Resonance Imaging for Myocardial Perfusion Assessment in Coronary Artery Disease Trial). Journal of Cardiovascular Magnetic Resonance, 14, 61. https://doi.org/10.1186/1532-429X-14-61

[20] Berman, D.S., Hachamovitch, R., Kiat, H., Cohen, I., Cabico, J.A., Wang, F.P., Friedman, J.D., Germano, G., Train, K.V. and Diamond, G.A. (1995) Incremental Value of Prognostic Testing in Patients with Known or Suspected Ischemic Heart Disease: A Basis for Optimal Utilization of Exercise Technetium-99 m Sestamibi Myocardial Perfusion SPECT. Journal of the American College of Cardiology, 26, 639-647. https://doi.org/10.1016/0735-1097(95)00218-S

[21] Sakuma, H., Suzawa, N., Ichikawa, Y., Makino, K., Hirano, T., Kitagawa, K. and Takeda, K. (2005) Diagnostic Accuracy of Stress First-Pass Contrast-Enhanced Myocardial Perfusion MRI Compared with Stress Myocardial Perfusion Scintigraphy. American Journal of Roentgenology, 185, 95-102. https://doi.org/10.2214/ajr.185.1.01850095

[22] Kurata, A., Kawaguchi, N., Kido, T., Inoue, K., Suzuki, J., Ogimoto, A., Funada, J., Higaki, J., Miyagawa, M., Vembar, M. and Mochizuki, T. (2013) Qualitative and Quantitative Assessment of Adenosine Triphosphate Stress Whole-Heart Dynamic Myocardial Perfusion Imaging Using 256-Slice Computed Tomography. PLoS ONE, 8, e83950. https://doi.org/10.1371/journal.pone.0083950

[23] Hachamovitch, R., Berman, D.S., Shaw, L.J., Kiat, H., Cohen, I., Cabico, J.A., Friedman, J. and Diamond, G.A. (1998) Incremental Prognostic Value of Myocardial Perfusion Single Photon Emission Computed Tomography for the Prediction of Cardiac Death: Differential Stratification for Risk of Cardiac Death and Myocardial Infarction. Circulation, 97, 535-543. https://doi.org/10.1161/01.CIR.97.6.535

[24] Le, H., Wong, J.T. and Molloi, S. (2008) Estimation of Regional Myocardial Mass at Risk Based on Distal Arterial Lumen Volume and Length Using 3D Micro-CT Images. Computerized Medical Imaging and Graphics, 32, 488-501. https://doi.org/10.1016/j.compmedimag.2008.05.002

[25] Termeer, M., Bescós, J.O., Breeuwer, M., Vilanova, A., Gerritsen, F., Groller, M.E. and Nagel, E. (2010) Patient-Specific Mappings between Myocardial and Coronary Anatomy. Dagstuhl Follow Ups, 1, 196-209.

[26] Kang, S.J., Yang, D.H., Kweon, J., Kim, Y.H., Lee, J.G., Jung, J., Kim, N., Mints, G.S., Kang, J.W., Lim, T.H. and Park, S.W. (2016) Better Diagnosis of Functionally Signify Intermediate Sized Narrowing Using Intravascular Ultrasound-Minimal Lumen Area and Coronary Computed Tomographic Angiography-Based Myocardial Segmentation. American Journal of Cardiology, 117, 1282-1288. https://doi.org/10.1016/j.amjcard.2016.01.022

[27] Tamarappoo, B.K., Gutstein, A., Cheng, V.Y., Nakazato, R., Gransar, H., Dey, D., Thomson, L.E., Hayes, S.W., Friedman, J.D., Germano, G., Slomka, P.J. and Berman, D.S. (2010) Assessment of the Relationship between Stenosis Severity and Distribution of Coronary Artery Stenosis on Multislice Computed Tomographic Angiography and Myocardial Ischemia Detected by Single Photon Emission Computed Tomography. Journal of Nuclear Cardiology, 17, 791-802.

https://doi.org/10.1007/s12350-010-9230-6 
[28] Korosoglou, G., Mueller, D., Lehrke, S., Steen, H., Hosch, W., Heye, T., Kauczor, H.U., Giannitsis, E. and Katus, H.A. (2010) Quantitative Assessment of Stenosis Severity and Atherosclerotic Plaque Composition Using 256-Slice Computed Tomography. European Radiology, 20, 1841-1850.

https://doi.org/10.1007/s00330-010-1753-3

Submit or recommend next manuscript to SCIRP and we will provide best service for you:

Accepting pre-submission inquiries through Email, Facebook, LinkedIn, Twitter, etc. A wide selection of journals (inclusive of 9 subjects, more than 200 journals)

Providing 24-hour high-quality service

User-friendly online submission system

Fair and swift peer-review system

Efficient typesetting and proofreading procedure

Display of the result of downloads and visits, as well as the number of cited articles

Maximum dissemination of your research work

Submit your manuscript at: http://papersubmission.scirp.org/

Or contact ojrad@scirp.org 